\title{
Medizinischer Zusatznutzen von Hämodiafiltration gegenüber Hämodialyse bezüglich Mortalität und Lebensqualität
}

\author{
Eine systematische Übersicht \\ D. Panteli ${ }^{* 1}$, F. Wittenbecher ${ }^{*}$, R. Busse ${ }^{1}$
}

Institute

1 Fachgebiet Management im Gesundheitswesen,

Technische Universität Berlin

*DP und FW haben zu gleichen Teilen zur Arbeit beigetragen.
Zusatzmaterial online einsehbar unter http://dx.doi.org/ 10.1055/s-0041-102110

\section{Korrespondenz} Dimitra Panteli Fachgebiet Management im Gesundheitswesen Technische Universität Berlin Sekretariat $\mathrm{H} 80$ Straße des 17. Juni 135 10623 Berlin Tel.: +493031428420 dimitra.panteli@tu-berlin.de

\section{Zusammenfassung}

Hintergrund | Bei Patienten mit terminalem chronischem Nierenversagen ist eine dauerhafte Nierenersatztherapie überlebensnotwendig. $\mathrm{Ob}$ Hämodiafiltration (HDF) der Hämodialyse (HD) hierbei überlegen ist, ist unklar. Ziel der vorliegenden Übersichtsarbeit ist es daher, zu evaluieren, ob HDF im Vergleich zu HD zu besseren Ergebnissen hinsichtlich Mortalität und Lebensqualität führt.

Methoden I Es wurde eine systematische Literatursuche durchgeführt. Die identifizierten Studien wurden methodisch bewertet und hinsichtlich ihrer Evidenz systematisch erfasst. Alle Arbeitsschritte fanden im September und Oktober 2013 statt.

Ergebnisse I Es wurden insgesamt 14 relevante Studien identifiziert. Drei davon waren groß, ran- domisiert und kontrolliert. Eine dieser Studien berichtete über eine signifikant niedrigere Mortalität im HDF-Arm, während in den beiden anderen keine signifikanten Unterschiede gefunden wurden. Eine große randomisierte kontrollierte Studie beinhaltete eine Analyse zur Lebensqualität. Dabei wurden keine signifikanten Unterschiede gefunden. Die übrige Evidenz sowohl für Mortalität als auch für Lebensqualität war verzerrungsanfällig und ihre Aussagekraft wurde daher als niedrig eingestuft.

Folgerung I Nur eine große randomisierte kontrollierte Studie zeigte eine statistisch signifikant niedrigere Mortalität bei Behandlung mit HDF für alle Behandelten. Von einem günstigen Einfluss der HDF auf das Gesamtüberleben ist auszugehen, sofern hohe Konvektionsvolumina erreicht werden. Für eine bessere Lebensqualität bei Behandlung mit HDF im Vergleich zu HD besteht keine verlässliche Evidenz.

\section{Einleitung}

Unterschiedliche Grunderkrankungen können zu dauerhaften Funktionseinschränkungen der Nieren führen - dem chronischen Nierenversagen. Häufigsten Ursachen des chronischen Nierenversagens in Deutschland sind

- Diabetes mellitus,

- Bluthochdruck und

- Glomerulonephritiden [7].

Im terminalen Stadium des chronischen Nierenversagens (engl. end stage renal disease, ESRD) ist eine dauerhafte Nierenersatztherapie (NET) notwendig [6].

Eine unzureichende Nierenfunktion bedeutet eine erhebliche Einschränkung der Lebenserwartung und in besonderem Maße auch der Lebensqualität. Die klinische Symptomatik reicht hier von psychischen Störungen über Knochenschmerzen bis hin zu Herzrhythmusstörungen $[6,15,21]$. Der tatsächliche Ersatz der Niere mit eingeschränkter Funktion durch eine Spenderniere erfolgt in einer geringeren Anzahl als theoretisch möglich. Grund hierfür ist in erster Linie ein Mangel an Spenderorganen [7].
Falls die Nierentransplantation nicht möglich ist, stehen Verfahren der NET zur Verfügung. In absteigender Häufigkeit sind dies in erster Linie

- die intermittierende Hämodialyse (HD, basierend auf Diffusion),

- die intermittierende Hämodiafiltration (HDF, basierend auf Diffusion und Konvektion) und

- die ambulante Peritonealdialyse (PD) [8, 23].

Die apparativen Verfahren HD und HDF sind für einen relativ großen und ähnlichen Kreis chronisch niereninsuffizienter Patienten als intermittierende NET prinzipiell geeignet [22]. In - sKasten 1 werden die Grundzüge beider Verfahren erläutert.

Die high-flux-HD (hf-HD, s. • sKasten 1) ist das derzeit am häufigsten verwendete Verfahren und kann somit als Standardverfahren betrachtet werden. Es bestehen erhebliche Forschungsbemühungen, die apparative NET zu optimieren. HDF gilt hierbei seit längerer Zeit als vielversprechende Weiterentwicklung. Die Evidenzlage hierzu ist insgesamt jedoch uneinheitlich. Das Ziel der vorliegenden systematischen Übersichtsarbeit war daher zu untersuchen, welchen Einfluss die HDF im Vergleich zur HD auf das Gesamtüberleben und die Mortalität sowie die 
Lebensqualität bei dauerhaft nierenersatzpflichtigen Patienten hat.

\section{Methoden}

Für die vorliegende Übersichtsarbeit wurde eine systematische Literatursuche in den Datenbanken PubMed und EMBASE durchgeführt. Die entsprechenden Suchstrategien sind in der $\$ sTab. 1 aufgeführt. Die öffentlich zugänglichen Studienregister des U.S. National Institute of Health (ClinicalTrials.gov) und der Weltgesundheitsorganisation (International Clinical Trials Registry Platform) wurden anhand einer vereinfachten Suchstrategie durchsucht. Die Referenzlisten erfasster systematischer Übersichtsarbeiten [24, $25,29,31]$ und in die Auswertung aufgenommener Primärstudien wurden genutzt, um weitere relevante Zitate zu identifizieren. Die in der Literatursuche identifizierten Treffer wurden anhand von vorbestimmten Einschlusskriterien bewertet ( $\triangleright$ sTab. 2). Die Literatursuche sowie die Bewertung und Synthese der erfassten Evidenz wurden zwischen September und Oktober 2013 durchgeführt, die letzte Aktualisierung der Suche erfolgte am 15. Oktober 2013.

Inhalte der eingeschlossenen Primärstudien wurden mittels eines standardisierten Extraktionsformulars in Anlehnung an Beispiele der Cochrane Collaboration [5] gewonnen. Um das Verzerrungspotenzial der individuellen Studien zu bewerten, wurde für randomisierte, kontrollierte Studien (RCT) das von der Cochrane Collaboration entwickelte Risk of Bias Tool [4] verwendet. Für Beobachtungsstudien kam eine angepasste Checkliste zum Einsatz. Verzerrungen sind systematische Veränderungen der Unterschiede zwischen den Vergleichsgruppen und führen dadurch zu geringerer Ergebnissicherheit. Sie sind in der Regel durch das Design oder die Durchführung der Studie bedingt. Studien, deren Verzerrungspotenzial als hoch eingestuft wurde, wurden nicht ausgeschlossen, sondern werden entsprechend in der Diskussion besprochen.

Zwei unabhängige Gutachter überprüften die Recherche-Treffer, bewerteten die Studien und extrahierten die relevanten Inhalte.

\section{Ergebnisse}

\section{Literaturrecherche}

Der Prozess der Studiensuche und -selektion wird als Flussdiagramm entsprechend der PRISMAEmpfehlungen in $\triangleright$ sAbb. 1 dargestellt [16]. Durch die verschiedenen Schritte wurden insgesamt 14 Studien (15 Publikationen) identifiziert, die der Forschungsfrage entsprachen. Die Übersicht der Studien wird mit Angaben zu
- Publikationsjahr,

- Studiendesign,

- Prüf- und Vergleichsintervention,

- Patientenanzahl und

- erfassten Endpunkten

in der $\checkmark$ Tab. 1 präsentiert. Gründe für den Ausschluss von Publikationen in der Volltextanalyse sind in sTab. 3 aufgeführt.

\section{Charakteristika der in die Bewertung eingeschlossenen Studien}

Bei 3 Studien wurde das Verzerrungspotenzial als niedrig eingestuft. Die übrigen eingeschlossenen Studien wiesen entweder Aspekte auf, die zu einer Verzerrung führen könnten, oder hatten ein unklares Verzerrungsrisiko. Die sTab. 4 zeigt das Verzerrungspotenzial der eingeschlossenen RCTs gemäß des passenden Instrumentes der Cochrane Collaboration [4].

Die Baseline-Charakteristika des Patientenkollektivs der 3 großen RCTs können der $\triangleright$ sTab. $\mathbf{5}$ entnommen werden. In keiner der 3 Studien wurden signifikante Unterschiede in den aufgeführten Parametern zwischen dem Interventionsund dem Kontrollarm berichtet. Sowohl in der CONTRAST- als auch in der türkischen HDF-Studie waren mehr Diabetes-Patienten im Interventionsarm, während es sich in der ESHOL-Studie umgekehrt verhielt. In letzterer waren die Patienten im Interventionsarm durchschnittlich um 1,8 Jahre jünger.

\section{Patientenrelevante Endpunkte}

Mortalität und Überleben | $>$ Tab. 2 und $\$ sTab. 6 und $\triangleright$ sTab. 7 fassen die Aussagen der eingeschlossenen Studien zu Mortalität und Gesamtüberleben zusammen. Effektschätzer werden hier so aufgeführt, wie sie in den Publikationen berichtet werden.

Für die Endpunkte Mortalität und Überleben wurden 3 große RCTs identifiziert $(\checkmark$ Tab. 2). Diese zeigten heterogene Ergebnisse. In der niederländischen CONTRAST-Studie [9] wurde die HDF mit der lf-HD verglichen. Die beiden anderen RCTs, die ESHOL-Studie [14] und die türkische HDF-Studie, [18] nutzten die hf-HD als Vergleichsintervention.

Die spanische ESHOL-Studie [14] zeigte ein statistisch signifikant um 30\% geringeres Risiko für Gesamtmortalität unter Behandlung mit HDF im Vergleich zur Behandlung mit hf-HD (HR 0,70; 95\%-KI [0,53-0,92;] p=0,01). CONTRAST (HR $0,95 ;[0,75-1,20])$ und die türkische HDF-Studie (HR 0,79; $[0,55-1,14])$ zeigten keinen statistisch signifikanten Vorteil eines der beiden untersuchten Verfahren bezogen auf die Gesamtmortalität. 


\begin{tabular}{|c|c|c|c|c|c|c|}
\hline Referenz & $\begin{array}{l}\text { Studien- } \\
\text { design }\end{array}$ & $\begin{array}{l}\text { Verfahren und } \\
\text { Patientenzahl* }\end{array}$ & $\begin{array}{l}\text { Studien-/ Nach- } \\
\text { beobachtungsdauer }\end{array}$ & $\begin{array}{l}\text { Relevante } \\
\text { Endpunkte }\end{array}$ & Ergebnis & Finanzierung \\
\hline $\begin{array}{l}\text { ESHOL } 2013 \\
{[14]}\end{array}$ & RCT & $\begin{array}{l}\text { ol-HDF }=456 \\
\mathrm{hf}-\mathrm{HD}=450\end{array}$ & 36 Monate & Mortalität (HR) & $\begin{array}{l}\text { signifikante Steigerung } \\
\text { des Gesamtüberlebens } \\
\text { unter ol-HDF um 30\% }\end{array}$ & $\begin{array}{l}\text { Catalan Society of } \\
\text { Nephrology, Fresenius } \\
\text { Medical Care, Gambro }\end{array}$ \\
\hline $\begin{array}{l}\text { Türkische } \\
\text { HDF-Studie } \\
2012 \text { [18] }\end{array}$ & $\mathrm{RCT}$ & $\begin{array}{l}\text { ol-HDF = 391 } \\
\text { hf-HD=391 }\end{array}$ & $\begin{array}{l}\text { Minimum: } \\
24 \text { Monate } \\
\text { Maximum: } \\
39 \text { Monate }\end{array}$ & Mortalität (HR) & $\begin{array}{l}\text { kein signifikanter } \\
\text { Unterschied in } \\
\text { Mortalität }\end{array}$ & $\begin{array}{l}\text { European Nephrology } \\
\text { and Dialysis Institute, } \\
\text { durchgeführt in } \\
\text { Fresenius Medical Care } \\
\text { Zentren }\end{array}$ \\
\hline $\begin{array}{l}\text { CONTRAST } \\
2012[9,15]\end{array}$ & $\mathrm{RCT}$ & $\begin{array}{l}\text { ol-HDF }=358 \\
\text { If-HD }=356\end{array}$ & 36 Monate & $\begin{array}{l}\text { Mortalität (HR) und } \\
\text { Lebensqualität } \\
\text { (KDQOL-SF) }\end{array}$ & $\begin{array}{l}\text { keine signifikanten } \\
\text { Unterschiede in } \\
\text { Mortalität oder } \\
\text { Lebensqualität }\end{array}$ & $\begin{array}{l}\text { Dutch Kidney } \\
\text { Foundation, Fresenius } \\
\text { Medical Care, Gambro } \\
\text { Lundia und andere }\end{array}$ \\
\hline $\begin{array}{l}\text { Locatelli et.al } \\
2010 \text { [13] }\end{array}$ & $\mathrm{RCT}$ & $\begin{array}{l}\mathrm{HDF}=40 \\
\text { If-HD }=70\end{array}$ & 24 Monate & $\begin{array}{l}\text { Mortalität } \\
\text { (Überlebenskurve) }\end{array}$ & $\begin{array}{l}\text { kein signifikanter } \\
\text { Unterschied in } \\
\text { Mortalität }\end{array}$ & k. A. \\
\hline $\begin{array}{l}\text { Wizemann et } \\
\text { al. } 2000 \text { [34] }\end{array}$ & $\mathrm{RCT}$ & $\begin{array}{l}\mathrm{HDF}=23 \\
\text { If-HD }=21\end{array}$ & 24 Monate & $\begin{array}{l}\text { Mortalität } \\
\text { (Zahlenangabe) }\end{array}$ & $\begin{array}{l}\text { kein signifikanter } \\
\text { Unterschied in } \\
\text { Mortalität }\end{array}$ & k. A. \\
\hline $\begin{array}{l}\text { Ward et al. } \\
2000 \text { [33] }\end{array}$ & RCT & $\begin{array}{l}\mathrm{HDF}=24 \\
\mathrm{hf}-\mathrm{HD}=21\end{array}$ & 12 Monate & $\begin{array}{l}\text { Lebensqualität } \\
\text { (KDQ) }\end{array}$ & $\begin{array}{l}\text { signifikante Verbesse- } \\
\text { rung physischer } \\
\text { Symptome }\end{array}$ & k. A. \\
\hline $\begin{array}{l}\text { Locatelli et.al } \\
1996 \text { [12] }\end{array}$ & RCT & $\begin{array}{l}\mathrm{HDF}=50 \\
\text { hf- } \mathrm{HD}=51 \\
\text { lf-HD }=54\end{array}$ & $\begin{array}{l}12 \text { Monate, dann } \\
\text { verlängert um } \\
\text { weitere } 12 \text { Monate }\end{array}$ & Mortalität & $\begin{array}{l}\text { keine Unterschiede in } \\
\text { Mortalität }\end{array}$ & k. A. \\
\hline $\begin{array}{l}\text { Kantartzi et al. } \\
2013[10]\end{array}$ & $\begin{array}{l}\text { Cross- } \\
\text { over }\end{array}$ & $\begin{array}{l}\text { ol-HDF } / \text { HDF- } \\
w b=48 / 44 \\
\mathrm{HD}=48 / 44\end{array}$ & $\begin{array}{l}4 \times 3 \text { Monate pro } \\
\text { Verfahren }\end{array}$ & $\begin{array}{l}\text { Lebensqualität } \\
\text { (SF-36) }\end{array}$ & $\begin{array}{l}\text { signifikanten } \\
\text { Unterschiede in } \\
\text { Lebensqualität zw. HD } \\
\text { u. ol-HDF / HDF }\end{array}$ & k. A. \\
\hline $\begin{array}{l}\text { Schiffl } 2007 \\
\text { [27] }\end{array}$ & $\begin{array}{l}\text { Cross- } \\
\text { over }\end{array}$ & $\begin{array}{l}\text { ol-HDF }=38 \\
h f-H D=38\end{array}$ & $\begin{array}{l}2 \times 24 \text { Monate pro } \\
\text { Verfahren }\end{array}$ & $\begin{array}{l}\text { Lebensqualität } \\
\text { (KDQ) }\end{array}$ & $\begin{array}{l}\text { Nachhaltige Verbesse- } \\
\text { rung physischer } \\
\text { Symptome bei ol-HDF }\end{array}$ & k. A. \\
\hline $\begin{array}{l}\text { RISCAVID } 2008 \\
{[20]}\end{array}$ & Kohorte & $\begin{array}{l}\text { ol-HDF }=129 \\
\text { HDF-wb }=204 \\
H D=424 \\
(I f=403, h f=21)\end{array}$ & 30 Monate & Mortalität (RR) & $\begin{array}{l}\text { signifikante Steige- } \\
\text { rung der Überlebens- } \\
\text { rate in HDF }\end{array}$ & Staatlich \\
\hline $\begin{array}{l}\text { Bosch et al. } \\
2006 \text { [2] }\end{array}$ & Kohorte & unklar & maximal 72 Monate & Mortalität (SMR) & $\begin{array}{l}\text { signifikant niedrigere } \\
\text { SMR unter HDF im } \\
\text { Vergleich zu USRDS- } \\
\text { Daten }\end{array}$ & k. A. \\
\hline $\begin{array}{l}\text { DOPPS II } 2006 \\
\text { [3] }\end{array}$ & Kohorte & $\begin{array}{l}\text { le-HDF = } 156 \\
\text { he-HDF }=97 \\
\text { If-HD = } 1366 \\
\text { hf-HD = } 546\end{array}$ & $\begin{array}{l}\text { ungenau, ca. } 36 \\
\text { Monate }\end{array}$ & $\begin{array}{l}\text { Mortalität (RR) und } \\
\text { Lebensqualität } \\
\text { (SF-36) }\end{array}$ & $\begin{array}{l}\text { signifikante Steigerung } \\
\text { der Überlebensrate bei } \\
\text { high-efficiency HDF, } \\
\text { keine Unterschiede in } \\
\text { der Lebensqualität }\end{array}$ & Amgen, Kirin \\
\hline $\begin{array}{l}\text { Knezevic et al. } \\
2012 \text { [11] }\end{array}$ & $\begin{array}{l}\text { Quer- } \\
\text { schnitt }\end{array}$ & $\begin{array}{l}\mathrm{HDF}=45 \\
\mathrm{hf}-\mathrm{HD}=39 \\
\text { If- } \mathrm{HD}=40\end{array}$ & $\mathrm{n} / \mathrm{a}$ & $\begin{array}{l}\text { Lebensqualität } \\
\text { (SF-36) }\end{array}$ & $\begin{array}{l}\text { signifikante Unter- } \\
\text { schiede in Lebens- } \\
\text { qualität zw. HDF und } \\
\text { hf-HD/ HDF und If-HD }\end{array}$ & Staatlich \\
\hline $\begin{array}{l}\text { Moreno et al. } \\
1996 \text { [17] }\end{array}$ & $\begin{array}{l}\text { Quer- } \\
\text { schnitt }\end{array}$ & $\begin{array}{l}1013 \text { Patienten } \\
\mathrm{HD}=88 \% \\
\mathrm{HDF}=7 \%\end{array}$ & $\mathrm{n} / \mathrm{a}$ & $\begin{array}{l}\text { Lebensqualität (KS, } \\
\text { SIP) }\end{array}$ & $\begin{array}{l}\text { kein Unterschied in } \\
\text { QoL nach Adjustierung }\end{array}$ & $\begin{array}{l}\text { P.E.N.S.A - Esteve } \\
\text { Stiftung }\end{array}$ \\
\hline
\end{tabular}

* es werden nur relevante Studienarme aufgeführt; HD: Hämodialyse; HDF: Hämodiafiltration; HDF-wb: HDF with bags; hf-HD: high-flux HD; HR: Hazard Ratio; KDQ: Kidney Disease Questionnaire; KDQOL-SF: Kidney Disease Quality of Life Short Form Fragebogen; KS: Karnofsky Scale; If-HD: low-flux HD; ol-HDF: online HDF; RCT: randomized controlled trial; SIP: Sickness Impact Profile; SF-12: Short-Form-12 Fragebogen; SF-36: Short-Form-36 Fragebogen; SMR: standardized mortality rate

Tab. 1 Studiencharakteristika 
In allen 3 Studien zeigten Subgruppenanalysen, dass diejenigen Patienten, bei denen im Durchschnitt die höchsten Konvektionsvolumina in der HDF erreicht wurden, den höchsten Überlebensvorteil hatten $(\checkmark$ Tab. 2 , fett hervorgehoben). Auch in der CONTRAST-Studie und in der türkischen HDF-Studie, in denen sich für die Gesamtheit der Patienten kein signifikanter Unterschied zeigte, ergaben Subgruppenanalysen eine statistisch signifikant niedrigere Mortalität für Patienten mit höheren Konvektionsvolumina (HR=0,62; [0,41-0,93]; HR=0,54; [0,33-0,88], jeweils unadjustierte Schätzer für das höchste Volumenstratum).

Zu beachten ist, dass die türkische HDF-Studie das Substitutions- und nicht das Konvektionsvolumen berichtete, im Gegensatz zu den beiden anderen Studien. Beim Konvektionsvolumen wird zusätzlich zu dem Volumen der substituierten Flüssigkeit (Substitutionsvolumen) der Gewichtsverlust des Patienten während der Dialysebehandlung berücksichtigt. In allen 3 Studien wurden die Subgruppenanalysen retrospektiv durchgeführt und müssen daher aus methodischer Sicht vorsichtig interpretiert werden.
In 3 weiteren RCTs $[12,13,34]$ mit hohem Verzerrungspotenzial, deutlich kleinerer Teilnehmerzahl und kürzerer Studiendauer wurden keine signifikanten Unterschiede in der Mortalität zwischen den Interventionsgruppen berichtet $(\triangleright$ sTab. 6).

2 größere Beobachtungsstudien, die RISCAVIDStudie [20] und die DOPPS-II-Studie [3], hingegen ergaben eine signifikant geringere Mortalität unter HDF. In der DOPPS-II-Studie war die geringere Mortalität wiederum abhängig von der Größe des Substitutionsvolumens (Ergebnisse aller Beobachtungsstudien zusammengefasst in $>$ sTab. 7).

Lebensqualität | In 4 Studien mit hohem Verzerrungspotenzial (1 RCT, 2 Studien mit CrossoverDesign und 1 Querschnittstudie) gab es Anhaltspunkte für eine bessere Lebensqualität unter HDF im Vergleich zur Kontrollintervention [10, 11, 27, 33]. Es zeigten sich insbesondere bessere Werte im Bereich „körperliche Symptome“. Eine dieser Studien [27] hatte eine Studiendauer von $2 \times 24$ Monaten, die restlichen 3 Studien nur eine Studiendauer von 12 Monaten oder weniger.

Die CONTRAST-Studie hatte das geringste Verzerrungspotenzial unter den Studien, die (auch) die
Tab. 2 Ergebnisse Mortalität große RCTs

\begin{tabular}{|c|c|c|c|c|c|c|c|c|}
\hline \multirow[t]{2}{*}{ Studie } & \multirow[t]{2}{*}{ Vergleich } & CV & SV & HR & & & & \\
\hline & & \multicolumn{2}{|c|}{ Liter pro Behandlung } & Berechnung & $\mathrm{HR}^{*}$ & KI-95\% & a) p-Wert & $\begin{array}{l}\text { b) p-Wert } \\
\text { Trend Test }\end{array}$ \\
\hline \multirow{4}{*}{$\begin{array}{l}\text { ESHOL } 2013 \\
{[14]}\end{array}$} & \multirow{4}{*}{ hf-HD } & Gesamt & & Unbereinigt & 0,70 & $0,53-0,92$ & 0,01 & \multirow{4}{*}{0,001} \\
\hline & & $<23,1$ & & Unbereinigt & 0,9 & $0,61-1,31$ & & \\
\hline & & $23,1-25,4$ & & Unbereinigt & 0,6 & $0,39-0,90$ & & \\
\hline & & $>25,4$ & & Unbereinigt & 0,55 & $0,34-0,84$ & & \\
\hline \multirow{7}{*}{$\begin{array}{l}\text { Türkische HDF-Studie } \\
2012 \\
{[18]}\end{array}$} & \multirow{7}{*}{ hf-HD } & & Gesamt & Unbereinigt & 0,79 & $0,55-1,14$ & 0,21 & \\
\hline & & \multirow{3}{*}{\multicolumn{2}{|c|}{$\leq 17,4$}} & Unbereinigt & 0,99 & $0,64-1,53$ & 0,54 & \\
\hline & & & & Model 1 & 1,17 & $0,73-1,88$ & 0,36 & \\
\hline & & & & Model 2 & 1,1 & $0,68-1,76$ & 0,69 & \\
\hline & & \multirow{3}{*}{\multicolumn{2}{|c|}{$>17,4$}} & Unbereinigt & 0,54 & $0,33-0,88$ & 0,01 & \\
\hline & & & & Model 1 & 0,57 & $0,33-0,96$ & 0,04 & \\
\hline & & & & Model 2 & 0,54 & $0,31-0,93$ & 0,02 & \\
\hline \multirow{10}{*}{$\begin{array}{l}\text { CONTRAST } 2012 \\
\text { [9] }\end{array}$} & \multirow{10}{*}{ If-HD } & Gesamt & & Unbereinigt & 0,95 & $0,75-1,20$ & & \\
\hline & & \multirow{3}{*}{\multicolumn{2}{|c|}{$<18,17$}} & Unbereinigt & 0,95 & $0,66-1,38$ & & \multirow{9}{*}{$\begin{array}{l}\text { Unbereinigt: } \\
\mathbf{0 , 0 1 0} \\
\text { Model 1: } \\
\mathbf{0 , 0 1 2} \\
\text { Model 2: } \\
\mathbf{0 , 0 1 5}\end{array}$} \\
\hline & & & & Model 1 & 0,79 & $0,53-1,14$ & & \\
\hline & & & & Model 2 & 0,8 & $0,52-1,24$ & & \\
\hline & & \multirow{3}{*}{\multicolumn{2}{|c|}{$18,18-21,95$}} & Unbereinigt & 0,83 & $0,57-1,22$ & & \\
\hline & & & & Model 1 & 0,77 & $0,51-1,14$ & & \\
\hline & & & & Model 2 & 0,84 & $0,54-1,29$ & & \\
\hline & & \multirow{3}{*}{\multicolumn{2}{|c|}{$>21,95$}} & Unbereinigt & 0,62 & $0,41-0,93$ & & \\
\hline & & & & Model 1 & 0,65 & $0,42-0,99$ & & \\
\hline & & & & Model 2 & 0,61 & $0,38-0,98$ & & \\
\hline
\end{tabular}

* Für die Vergleichsgruppe (hf-HD bzw. If-HD) gilt HR=1,0; p-Werte angegeben wie berichtet, bei freien Feldern wurde kein p-Wert angegeben, statistisch signifikante Werte durch Fettdruck hervorgehoben; a) Gruppenvergleich; b) Trend; CV: Convection Volume; HD: Hämodialyse; HDF: Hämodiafiltration; hf-HD: high-flux HD; If-HD: low-flux HD; HR: Hazard Ratio; RCT: Randomized Controlled Trial; RR: Relatives Risiko; SV: Substitutionsvolumen; weitere Informationen zu den statistischen Modellen sind in $\triangleright$ sTab. $\mathbf{5}$ enthalten. 
Lebensqualität untersucht haben ( sTab. 8). Hier konnte keine signifikante Verbesserung der Lebensqualität im Vergleich zur Kontrollintervention gezeigt werden. Subgruppenanalysen - unter anderem anhand des Konvektionsvolumens konnten hier keinen signifikanten Vorteil der HDF nachweisen. Auch die relativ große Beobachtungsstudie DOPPS II [3] hat keine bessere Lebensqualität unter HDF im Vergleich zu anderen Interventionen ergeben. Die Vergleichbarkeit der Studien, die die Lebensqualität untersucht haben, ist neben dem unterschiedlichen Verzerrungspotenzial auch dadurch eingeschränkt, dass unterschiedliche Messinstrumente genutzt wurden.

\section{Diskussion}

Es wurden 14 Studien in die systematische Übersichtsarbeit eingeschlossen. Hiervon hatten 3 Studien (alle RCTs) ein niedriges Verzerrungspotenzial. Von diesen 3 RCTs haben alle den Endpunkt Mortalität ausgewertet, nur eine Studie hat zusätzlich die Lebensqualität evaluiert.

Die Datenlage für Mortalität stellt sich heterogen dar. Während in der ESHOL-Studie eine relativ ausgeprägte Verbesserung der Mortalität unter HDF gezeigt wurde (30\% niedrigeres Risiko im Vergleich zu hf-HD), fanden die anderen beiden RCTs mit niedrigem Verzerrungspotenzial keinen statistisch signifikanten Unterschied zwischen den Gesamtgruppen. Im Vergleich zu den beiden anderen RCTs haben die Patienten in der ESHOLStudie insgesamt höhere Konvektionsvolumina erreicht. Dies ist insofern relevant, als dass Subgruppenanalysen aller drei Studien ergeben haben, dass das Konvektionsvolumen entscheidend für die Verbesserung der Mortalität ist.

Auffallend ist in dieser Hinsicht, dass in den vorliegenden Studien keine einheitlichen Definitionen für die unterschiedlichen Interventionen verwendet werden, wodurch die Vergleichbarkeit zusätzlich eingeschränkt wird. Dies gilt teilweise auch für die Vergleichsintervention HD, insbesondere aber für die HDF.

In Anbetracht der Tatsache, dass vor allem ein hohes Konvektionsvolumen vorteilhaft für die Patienten ist, muss also weiterhin eine Abgrenzung der hoch-effizienten oder hoch-volumigen HDF stattfinden. Hierfür scheinen die Begrifflichkeiten nicht eindeutig geregelt zu sein, obwohl Definitionen existieren [26]. Auch kann aus den vorliegenden Studien kein Grenzwert für das Konvektionsvolumen abgeleitet werden, ab welchem ein geringeres Mortalitätsrisiko wahrscheinlich ist, da sich die Subgruppenanalysen zwischen den 3 großen RCTs in der Gruppeneinteilung erheblich unterschieden.
Insgesamt ist die vorhandene Evidenz für einen Nutzen der HDF gegenüber HD bezogen auf Lebensqualität noch heterogener und weniger robust. Studien mit relativ niedrigem Verzerrungspotenzial zeigten keinen signifikanten Unterschied, auch nicht bei hohen Konvektionsvolumina. Lediglich kleinere und verzerrungsanfällige Studien deuteten darauf hin, dass auch in diesem Endpunkt eine Verbesserung unter HDF möglich wäre.

Für die vorliegende Übersichtarbeit wurden nur in Publikationen veröffentlichte Daten erfasst, auf Autorenanfragen wurde verzichtet. Somit konnten nicht-öffentliche Studienberichte nicht in die Bewertung miteinbezogen werden. Trotz dieser Einschränkung ist von einer umfassenden Darstellung der aktuell verfügbaren Evidenz zu HDF und HD auszugehen. Eine Meta-Analyse erscheint aufgrund der Heterogenität der Studien hinsichtlich

- Methodik,

- Intervention und Kontrolle,

- Endpunkten und

- Zeithorizont

nicht sinnvoll.

Zusammenfassend ist die Datenlage anhand von publizierten Primärstudien zu uneinheitlich und nicht ausreichend robust, um eindeutige Schlussfolgerungen über den relativen Einfluss von HDF auf Gesamtüberleben und gesundheitsbezogene Lebensqualität zu ziehen. Die HDF gilt als sicheres Verfahren [30]. In den vorliegenden Studien wurde allerdings kaum thematisiert, für welche Patientengruppe die HDF geeignet ist. Nur bei Erreichen hoher Konvektionsvolumina kann in Anbetracht der vorliegenden Evidenz ein Überlebensvorteil durch HDF vermutet werden. Aufgrund der vorliegenden Ergebnisse erscheint es wichtig, zu evaluieren, bei welchen Patienten ausreichend hohe Konvektionsvolumina erreicht werden können. Studien, die diese Fragestellung beantworten sollen, werden durchgeführt, wobei Ergebnisse noch ausstehen.

Konsequenz für Klinik und Praxis

- Die Hämodiafiltration bringt keine eindeutige Verbesserung der Gesamtmortalität im Vergleich zur Hämodialyse.

- Ein Zusatznutzen könnte aber erreicht werden, wenn während der Behandlung ein hoher Flüssigkeitsaustausch stattfindet.

- Es sind daher weitere Daten erforderlich, um das optimale Konvektionsvolumen zu finden und Patienten zu identifizieren, die von HDF profitieren können.

- Die Evidenzlage bezüglich Lebensqualität bei Hämodiafiltration ist uneinheitlich und nicht ausreichend für eine Bewertung. 


\section{Literatur}

1 Antonelli M, Azoulay E, Bonten M et al. Year in review in intensive care medicine 2009: I. Pneumonia and infections, sepsis, outcome, acute renal failure and acid base, nutrition and glycaemic control. Intens Care Med 2010; 36: 196-209

2 Bosch JP, Lew SQ, Barlee V et al. Clinical use of high-efficiency hemodialysis treatments: Long-term assessment. Hemodial Int 2006; 10: 73-81

3 Canaud B, Bragg-Gresham JL, Marshall MR et al. Mortality risk for patients receiving hemodiafiltration versus hemodialysis: European results from the DOPPS. Kidney Int 2006; 69: 2087-2093

4 Cochrane Collaboration, Cochrane Bias Methods Group. Research Projects: Cochrane Risk of Bias tool. Cochrane Collaboration 2013

5 Cochrane Hematological Malignancies Group . Ressourcen für Review Autoren 2013. http://chmg.cochrane.org/sites/chmg.cochrane.org/ files/uploads/Template-Data\%20Extraction-CHMG.pdf Letzter Zugriff 27.04.2015

6 Deutsche Arbeitsgemeinschaft für klinische Nephrologie e. V. Dialysestandard 2006. Deutsche Arbeitsgemeinschaft für klinische Nephrologie e. V., 2006

7 DGfN. Daten und Fakten zur Niere und zu Nierenersatzverfahren. Deutsche Gesellschaft für Nephrologie, 2013

8 Frei U, Schober-Halstenberg $\mathrm{H}$-J. Nierenersatztherapie in Deutschland - Bericht über Dialysebehandlung und Nierentransplantation in Deutschland 2006-2007. Berlin: Quasi-Niere gGmbH, 2008

9 Grooteman MP, van den Dorpel MA, Bots ML et al. Effect of online hemodiafiltration on all-cause mortality and cardiovascular outcomes. J Am Soc Nephrol 2012; 23: 1087-1096

10 Kantartzi K, Panagoutsos S, Mourvati E et al. Can dialysis modality influence quality of life in chronic hemodialysis patients? Low-flux hemodialysis versus high-flux hemodiafiltration: A cross-over study. Ren Fail 2013; 35: 216-221

11 Knezevic MZ, Djordjevic VV, Radovanovic-Velickovic RM. Influence of dialysis modality and membrane flux on quality of life in hemodialysis patients. Ren Fail 2012; 34: 849-855

12 Locatelli F, Mastrangelo F, Redaelli B et al. Effects of different membranes and dialysis technologies on patient treatment tolerance and nutritional parameters. The Italian Cooperative Dialysis Study Group. Kidney Int 1996; 50: 1293-1302.

13 Locatelli F, Altieri P, Andrulli S et al. Hemofiltration and hemodiafiltration reduce intradialytic hypotension in ESRD. J Am Soc Nephrol 2010; 21: 1798-1807

14 Maduell F, Moreso F, Pons M et al. High-efficiency postdilution online hemodiafiltration reduces all-cause mortality in hemodialysis patients. J Am Soc Nephrol 2013; 24: 487-497

15 Mazairac AH, de Wit GA, Grooteman MP et al. Effect of hemodiafiltration on quality of life over time. Clin J Am Soc Nephrol. 2013; 8: 82-89

16 Moher D, Liberati A, Tetzlaff J et al. Preferred reporting items for systematic reviews and meta-analyses: the PRISMA statement. PLoS Med 2009; 6: e1000097

17 Moreno F, Lopez Gomez JM, Sanz-Guajardo D et al. Quality of life in dialysis patients. A spanish multicentre study. Spanish Cooperative Renal Patients Quality of Life Study Group. Nephrol Dial Transplant 1996; 11 (Suppl. 2): 125-129

18 Ok E, Asci G, Toz H et al. Mortality and cardiovascular events in online haemodiafiltration (OL-HDF) compared with high-flux dialysis: results from the Turkish OL-HDF Study. Nephrol Dial Transplant 2013; 28: 192-202

19 Palmer SC, Rabindranath KS, Craig JC et al. High-flux versus low-flux membranes for end-stage kidney disease. Cochrane Db Syst Rev 2012; 9: CD005016

20 Panichi V, Rizza GM, Paoletti S et al. Chronic inflammation and mortality in haemodialysis: effect of different renal replacement therapies. Results from the RISCAVID study. Nephrol Dial Transplant 2008; 23: 2337-2343
21 Parmar MS. Chronic renal disease. BMJ 2002; 325: 85-90

22 Petrie J, $\mathrm{Ng}$ TG, Hawley CM. Review Article: is it time to embrace haemodiafiltration for centre-based haemodialysis? Nephrology. 2008; 13: 269-277

23 Potthoff F, Münscher C, Berendes A, Weber W. Jahresbericht Datenanalyse Dialyse für den Gemeinsamen Bundesausschuss Berichtsjahr: 2012. Münster: MNC-Medical Netcare GmbH, 15.03.2013

24 Rabindranath KS, Strippoli GF, Roderick P et al. Comparison of hemodialysis, hemofiltration, and acetate-free biofiltration for ESRD: systematic review. Am J Kidney Dis. 2005; 45: 437-447

25 Rabindranath KS, Strippoli GF, Daly C et al. Haemodiafiltration, haemofiltration and haemodialysis for end-stage kidney disease. Cochrane Db Syst Rev 2006; 4: CD006258

26 Ronco C. Evolution of hemodiafiltration. Contrib Nephrol 2007; 158: 9-19

27 Schiffl H. Prospective randomized cross-over long-term comparison of online haemodiafiltration and ultrapure high-flux haemodialysis. Eur J Med Res 2007; 12: 26-33

28 Schulz KF, Altman DG, Moher D et al. CONSORT 2010 Statement: updated guidelines for reporting parallel group randomised trials. Trials. 2010; 11: 32

29 Susantitaphong P, Siribamrungwong M, Jaber BL. Convective therapies versus low-flux hemodialysis for chronic kidney failure: a meta-analysis of randomized controlled trials. Nephrol Dial Transplant 2013; 28 : 2859-2874

30 Tattersall JE, Ward RA, group E. Online haemodiafiltration: definition, dose quantification and safety revisited. Nephrol Dial Transplant 2013; 28: 542-550

31 Varela Lema L, Ruano Ravina A. Effectiveness and safety of different hemodialysis modalities: a review. J Nephrol 2007; 20: 525-542

32 von Elm E, Altman DG, Egger M et al. The Strengthening the Reporting of Observational Studies in Epidemiology (STROBE) statement: guidelines for reporting observational studies. J Clin Epidemiol 2008; 61: 344-349

33 Ward RA, Schmidt B, Hullin J at al. A comparison of on-line hemodiafiltration and high-flux hemodialysis: a prospective clinical study. J Am Soc Nephrol 2000; 11: 2344-2350

34 Wizemann V, Lotz C, Techert F, Uthoff S. On-line haemodiafiltration versus low-flux haemodialysis. A prospective randomized study. Nephrol Dial Transplant 2000; 15 (Suppl. 1): 43-48

Interessenkonflikt

Die vorliegende Arbeit beruht auf einem Health Technology Assessment (HTA) zur hochvolumigen Hämodiafiltration bei Patienten mit chronischem Nierenversagen, das von der Fresenius Medical Care Deutschland GmbH finanziell unterstützt wurde. Die wissenschaftliche Unabhängigkeit beim Bearbeiten des HTA sowie beim Verfassen dieses Manuskriptes war zu jeder Zeit gegeben.

Das Fachgebiet Management im Gesundheitswesen nimmt an einer Reihe von drittmittelfinanzierten Forschungsaktivitäten teil. Die Durchführung der vorliegenden Arbeit war davon abgegrenzt und unabhängig.
DOI 10.1055/s-0041-102110 Dtsch Med Wochenschr 2015 140: e114-e119 (c) Georg Thieme Verlag KG . Stuttgart - New York . ISSN 0012-0472 